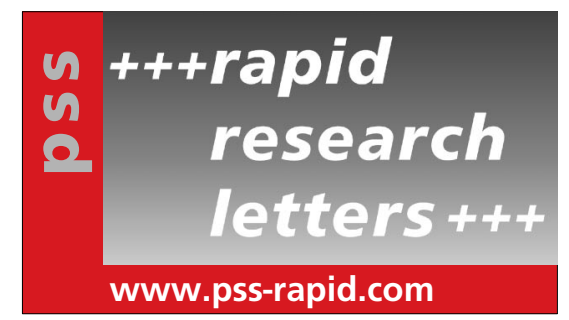

\title{
Efficient nanocoax-based solar cells
}

\author{
M. J. Naughton ${ }^{* 1,2}$, K. Kempa ${ }^{* * 1,2}$, Z. F. Ren ${ }^{* * * 1,2}$, Y. Gao', J. Rybczynski ${ }^{2}$, N. Argenti' ${ }^{2}$, W. Gao ${ }^{2}$, Y. Wang' \\ Y. Peng', J. R. Naughton', G. McMahon', M. J. Burns'², A. Shepard², M. Clary², C. Ballif ${ }^{3}$, F.-J. Haug ${ }^{3}$, \\ T. Söderström ${ }^{3}$, O. Cubero ${ }^{3}$, and C. Eminian ${ }^{3}$ \\ ${ }^{1}$ Department of Physics, Boston College, Chestnut Hill, Massachusetts 02467, USA \\ ${ }^{2}$ Solasta Inc., Newton, Massachusetts 02458, USA \\ ${ }^{3}$ École Polytechnique Fédérale de Lausanne (EPFL), Institute of Microengineering (IMT), 2000 Neuchatel, Switzerland
}

Received 17 April 2010, revised 29 May 2010, accepted 31 May 2010

Published online 4 June 2010

Keywords photovoltaics, solar cells, nanoscale, nanocoax

\footnotetext{
${ }^{*}$ Corresponding authors: e-mail naughton@bc.edu, Phone: (617) 552-0635, Fax: (617) 552-8478

** e-mail kempa@bc.edu, ${ }^{* * *}$ e-mail renzh@bc.edu
}

The power conversion efficiency of most thin film solar cells is compromised by competing optical and electronic constraints, wherein a cell must be thick enough to collect light yet thin enough to efficiently extract current. Here, we introduce a nanoscale solar architecture inspired by a well-known radio technology concept, the coaxial cable, that naturally resolves this "thick-thin" conundrum. Optically thick and elec- tronically thin amorphous silicon "nanocoax" cells are in the range of $8 \%$ efficiency, higher than any nanostructured thin film solar cell to date. Moreover, the thin nature of the cells reduces the Staebler-Wronski light-induced degradation effect, a major problem with conventional solar cells of this type. This nanocoax represents a new platform for low cost, high efficiency solar power.
1 Introduction Despite requiring thick layers of relatively costly material, the vast majority of today's solar photovoltaic (PV) cells employ crystalline media, due to their superior energy conversion efficiency compared to noncrystalline, "thin film" cells [1]. The dominant material, crystalline silicon (c-Si), has weak optical absorption, and so must be relatively thick $(\sim 200 \mu \mathrm{m})$ to efficiently collect light. However, with charge carrier (electron and hole) mean free paths comparable to this distance, high power conversion efficiency $\eta$ can still be achieved ( $\eta \sim 25 \%$ for singlejunction cells) [2]. Noncrystalline materials such as amorphous silicon (a-Si), on the other hand, are strongly absorbing, such that thin films (under $1 \mu \mathrm{m}$ ) suffice for efficient light collection. However, mean free paths in a-Si are significantly shorter $(\sim 100 \mathrm{~nm})$ than in $\mathrm{c}-\mathrm{Si}$, such that thin film efficiency $(\eta<10 \%)$ severely lags its crystalline counterpart $[3,4]$. Both types of solar cells are therefore compromised by a coupling of the optical and electronic length scales: crystalline in terms of cost, thin film in terms of efficiency. This "thick-thin" paradox is difficult to resolve in the conventional, planar solar cell configuration, where photons and electrons travel essentially in the same direction, i.e. normal to the cell surface. Here we propose to resolve this problem by employing a cell structure based on a coaxial cable.
2 The nanocoax Conceived by Thomson more than 150 years ago for the first successful transatlantic communication cable [5] (and for which he was knighted as Lord Kelvin), the now ubiquitous coaxial cable is well-known in radio technology as a subwavelength transmission line [6]. It consists of a metallic core, a cylindrical dielectric sleeve, and a cylindrical outer metal. Radiation can propagate in the dielectric sleeve of a coax as a transverse electromagnetic (TEM) mode, which is low loss and cut-off free (i.e. broadband).

Recently, some of us showed [7, 8] that visible light can also be propagated multi-wavelength distances along a nanocoaxial cable, in spite its subwavelength radial dimensions. The main idea of the present work is to employ an array of such optical nanocoaxes, now with the dielectric sleeve replaced by a photovoltaic absorber, as the foundation of a high efficiency solar cell. The TEM mode also propagates in the so-called multicore coax configuration, in which more than one coaxial core is present [9]. This fact allows for a related, distributed version of the nanocoax solar cell, starting with an array of parallel metallic nanopillars, which have been shown to function as highly efficient optical antennae [10]. In this design, the electrodynamic shielding role of the outer coax electrode 


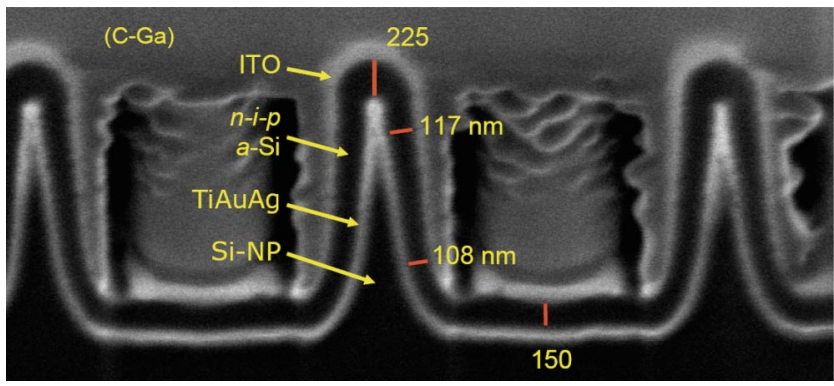

Figure 1 (online colour at: www.pss-rapid.com) Electron microscope image of a completed $\mathrm{n}-\mathrm{i}-\mathrm{p}$ a-Si nanocoax solar cell with only $\sim 90 \mathrm{~nm}$ i-layer thickness.

is replaced by the collective action of neighboring nanopillars.

Meanwhile, the electrostatic role (for charge extraction) can be taken by a transparent conductive coating. Each pillar, of course, is still coated with the PV absorber. Both versions of the nanocoax resolve the "thick-thin" problem: while the nanocoax can be made sufficiently long so that virtually all light is collected, the interelectrode gap (the PV thickness, measured radially) can be made small enough (subwavelength) to assure efficient carrier extraction. Light propagates along the pillars via a TEM-like mode, as confirmed by computer simulations [8, 11]. We have used several materials to define the starting nanopillar array, including carbon nanofibers [12], crystalline and amorphous silicon, silicon oxide, aluminum, and a variety of polymers.

3 Nanocoax solar cell While more straightforward to implement, this distributed version of the nanocoax can still encounter difficulties in the conformal coating of requisite films. We have thus developed a nanopillar shape that is optimized for conformality, while remaining a viable backbone for a functioning nanocoax. This shape, shown in Fig. 1, contains only smooth angular variations, with no sharp bends. The figure shows a focused ion beam (FIB)-induced secondary electron image of a completed nanocoax cell, with the constituent layers exposed via FIB milling. This nanocoax array was fabricated by etching a crystalline Si wafer, achieving a nanopillar (NP) array with site density $\sim 10^{8} / \mathrm{cm}^{2}$, and height $1.6 \mu \mathrm{m}$. It was subsequently sputter-coated with a $\mathrm{Ti}-\mathrm{Au}-\mathrm{Ag}$ film (8-4-30 nm thick, measured radially). These metallized nanopillars are then conformally coated with a-Si in an $\mathrm{n}-\mathrm{i}-\mathrm{p}$ configuration $(15-90-15 \mathrm{~nm}$ thick, respectively, at the midheight) via plasma-enhanced chemical vapor deposition, using $\mathrm{SiH}_{4}$ and $\mathrm{H}_{2}$ gases for the intrinsic/absorber (i-) layer, with $\mathrm{B}_{2} \mathrm{H}_{6}$ and $\mathrm{PH}_{3}$ gases additionally employed for $\mathrm{p}$ - and n-doping, respectively [13]. Indium tin oxide (ITO, $\sim 50 \mathrm{~nm}$ thick, radially), rf-sputtered from an ITO target, forms the top contact (the $\mathrm{C}-\mathrm{Ga}$ shown was deposited later to enable FIB sectioning). Uniquely among nanoscale approaches to solar energy conversion, such as core-shell semiconductor nano-wires [14-20] and bulk heterojunction cells [21], both majority and minority car-
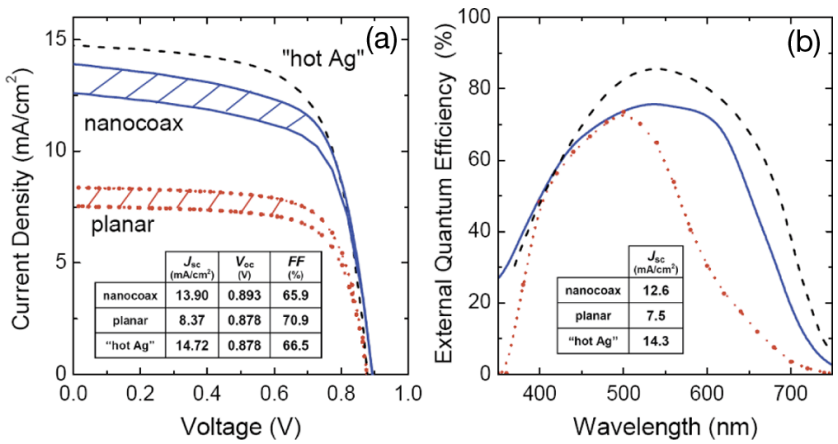

Figure 2 (online colour at: www.pss-rapid.com) Photovoltaic performance of the solar cells discussed in the text under AM1.5 simulated solar illumination. Power conversion efficiency is determined from $\eta=J_{\mathrm{sc}} V_{\mathrm{oc}} F F / P_{\mathrm{in}}$, where $J_{\mathrm{sc}}$ is the short circuit current density, $V_{\text {oc }}$ the open circuit voltage, $F F$ the fill factor, and $P_{\text {in }}$ the incident solar power density $\left(100 \mathrm{~mW} / \mathrm{cm}^{2}\right.$ for AM1.5). Upper and lower $J_{\text {sc }}$ ranges shown in the left panel are from transport and integrated EQE measurements (right panel), respectively. Total areas, measured with digitized optical microscopy, were used for all current density calculations.

riers in the nanocoax are delivered to proximate, metallic electrodes (the inner and outer conductors of the nanocoaxes), leading to low series resistance.

The photovoltaic performance of the nanocoax solar cell of Fig. 1, with the $90 \mathrm{~nm}$ average i-layer absorber thickness, is shown in Fig. 2. This thickness is determined by separate experiments calibrating the $n$ - and p-layer thicknesses, as well as the $\mathrm{p}+\mathrm{i}+\mathrm{n}$ thickness, all determined by transmission electron microscopy. It is compared to a planar device with the same absorber thickness, and to a textured, "hot" silver [22] solar cell with a much thicker absorber $(300 \mathrm{~nm})$. Current-voltage characteristics, PV parameters and corresponding external quantum efficiencies (EQE) are shown. The power conversion efficiency of the nanocoax cell $\left(0.18 \mathrm{~cm}^{2}\right.$ total area $)$ is $\eta=8.2 \%$ ( $\eta=8.4 \%$ active area efficiency), a remarkable fact for so thin a film. As demonstrated in Fig. 2, this performance well exceeds $(>50 \%$ higher) the efficiency of a planar cell $\left(0.12 \mathrm{~cm}^{2}\right)$ with similar thickness, $\eta=5.2 \%$, and approaches that of the high efficiency, textured cell $\left(0.12 \mathrm{~cm}^{2}\right)$ which has more than 3 times thicker absorber, $\eta=8.6 \%$. Cell areas are defined by lithographically prepared top contacts. Spectrum-integrated EQE-derived $J_{\text {sc }}$ values, and thus efficiencies, are smaller, but the relative performance of the individual cells is similar for both $J-V$ and EQE methods.

4 Reduced SWE Another significant advantage of this nanocoax cell follows from the fact that light-induced degradation of a-Si solar cells, via the Staebler-Wronski effect (SWE) [23], is known to diminish for ultrathin i-layers [24] such as those employed here. Our cells indeed show significantly reduced degradation, as shown in Fig. 3. Here, we plot the efficiencies of several conventional cells and two nanocoax a-Si cells, all normalized to their initial 


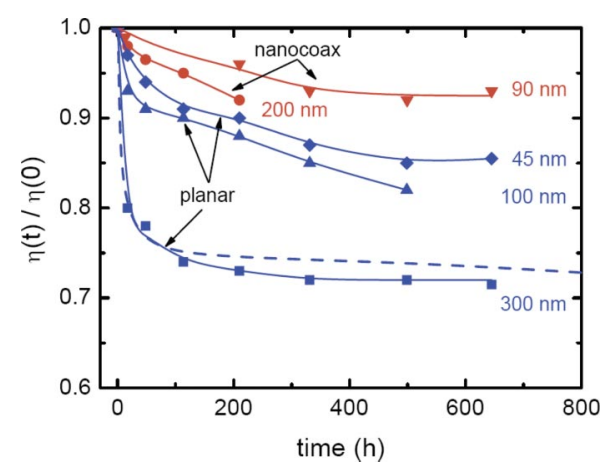

Figure 3 (online colour at: www.pss-rapid.com) Light-induced degradation of representative planar and nanocoax cells with various PV thicknesses. Note that the nanocoax cells have about half the degradation of their planar counterparts. Dashed line is Wronski's result for a conventional planar a-Si solar cell [25]. The $200 \mathrm{~nm}$ nanocoax sample was inadvertently damaged after 200 hours.

values, after prolonged soaking in AM1.5 light. It can be seen that a conventionally thick planar cell $(300 \mathrm{~nm})$ degrades significantly (by $\sim 25 \%$ ) in the first 50 hours or so, before saturating in the stabilized state. As expected, thinner planar cells (100 $\mathrm{nm}$ and $45 \mathrm{~nm}$ ) degrade less (note that these are too thin and thus too poorly absorbing to be efficient for practical use). However, note also that the $\eta>8 \%, 90 \mathrm{~nm}$-thick nanocoax cell degrades only $\sim 8 \%$ after $500 \mathrm{~h}$, a degradation half or less of that of the $100 \mathrm{~nm}$ and even the $45 \mathrm{~nm}$ thick planar cells. This is a remarkable result that follows directly from the nanocoax architecture: in addition to facilitating thinner films, this configuration spatially distributes incident light in such a way that the local intensity per unit volume is less than in a planar cell. Furthermore, the small degradation we still do observe could be explained by imperfect a-Si coating, with somewhat thicker PV near the tops of each nanocoax (see Fig. 1). Improved conformality may well reduce the SWE even further, and render even smaller the difference between initial and light-soaked values.

It is clear to us that our nanocoax, as fabricated, is not yet optimal. The preferred, ultimate solution is to increase the pillar length to over $2 \mu \mathrm{m}$, while at the same time reducing both the array pitch and the i-layer thickness. That is, absorbers even thinner than the present $90 \mathrm{~nm}$ are preferred, similar to that which we found in other work, where reducing a planar junction thickness dramatically improved the quality of the cell [26]. This requires a deposition technique capable of conformal deposition of PV absorbers, in particular a-Si, such as that provided by atomic layer deposition (ALD), which to our knowledge cannot be used for a-Si, at present.

Finally, we have also investigated shorter nanopillars $(\sim 600 \mathrm{~nm})$, in a configuration in which thicker PV films can be used, though at the expense of the nanocoax's optical advantage. With an a-Si i-layer absorber thickness of only $200 \mathrm{~nm}$ (and p- and n-layer thicknesses of 10-15 nm each), we have achieved initial efficiency $\sim 9 \%$, well above conventional untextured planar $(\eta \sim 6 \%)$ and $\mathrm{ZnO}-$ textured planar $(\eta \sim 7.5 \%)$ control cells prepared by us with matched film thicknesses (but still below the world records for initial or stabilized $\mathrm{p}-\mathrm{i}-\mathrm{n}$ a-Si [27]). This demonstrates the increased light absorption of even a quasinanocoax geometry. Further modifications to the nanocoax design may lead to yet higher efficiency while employing thinner than thin-film layers. Moreover, using, e.g. nanoimprint lithography, far less costly (polymer) replicated nanopillar substrates can be prepared.

Acknowledgements This work was supported by the US Department of Energy, contract number DE-FG36-08GO18013. It is based on the content of United States Patent No. 7,589,880.

\section{References}

[1] N. S. Lewis and G. Crabtree, Basic Research Needs for Solar Energy Utilization (US Department of Energy, 2005), www.er.doe.gov/bes/reports/abstracts.html\#SEU.

[2] M. A. Green et al., Prog. Photovolt. Res. Appl. 17, 85 (2009).

[3] M. Python et al., Sol. Energy Mater. Sol. Cells 93, 1714 (2009).

[4] B. Rech and H. Wagner, Appl. Phys. A 69, 155 (1999).

[5] W. Thomson, Philos. Mag. 9, 531 (1855); Proc. R. Soc. (London) 7, 382 (1855).

[6] D. Jackson, Classical Electrodynamics, 2nd ed. (Wiley, New York, 1975).

[7] J. Rybczynski, K. Kempa, A. Herczynski, Y. Wang, M. J. Naughton, Z. F. Ren, Z. P. Huang, D. Cai, and M. Giersig, Appl. Phys. Lett. 90, 021104 (2007).

[8] Y. Peng, X. Wang, and K. Kempa, Optics Express 16, 1758 (2008).

[9] D. M. Pozar, Microwave Engineering, 3rd ed. (Wiley, New York, 2005).

[10] Y. Wang et al., Appl. Phys. Lett. 85, 4741 (2004).

[11] K. Kempa, X. Wang, Z. F. Ren, and M. J. Naughton, Appl. Phys. Lett. 92, 043114 (2008).

[12] Z. F. Ren et al., Science 282, 1105 (1998).

[13] T. Söderström, F.-J. Haug, V. Terrazzoni-Daudrix, and C. Ballif, J. Appl. Phys. 103, 114509 (2008).

[14] B. Tian et al., Nature 449, 885 (2007).

[15] E. C. Garnett and P. Yang, J. Am. Chem. Soc. 130, 9224 (2008).

[16] Y. Zhang et al., Nano Lett. 7, 1264 (2007).

[17] Z. Fan, et al., Nano Res. 2, 829 (2009).

[18] J. Zhu et al., Nano Lett., DOI: 10.1021/n19034237 (2009).

[19] J. Fan et al., Nature Mater. 8, 648 (2009).

[20] J. A. Czaban et al., Nano Lett. 9, 148 (2009).

[21] G. Yu, J. Gao, J. C. Hummelin, F. Wudl, and A. J. Heeger, Science 270, 1789 (1995).

[22] A. Banerjee and S. Guha, J. Appl. Phys. 69, 1030 (1991).

[23] D. L. Staebler and C. R. Wronski, Appl. Phys. Lett. 31, 292 (1977).

[24] K. Zhu et al., Mater. Res. Soc. Symp. 762, 218 (2003).

[25] C. Wronski, Sol. Energy Mater. Sol. Cells 41/42, 427 (1996).

[26] K. Kempa, M. J. Naughton, Z. F. Ren, A. Herczynski, T. Kirkpatrick, J. Rybczynski, and Y. Gao, Appl. Phys. Lett. 95, 233121 (2009).

[27] S. Benagli et al., Proc. 24th EU PVSEC (2009), p. 2293. 